\title{
EQUIVALENCIA ENTRE SERIES TEMPORALES DE DIVERSIDAD PARA DOS NIVELES TAXONÓMICOS.
}

Alfredo Giraldo Mendoza ${ }^{1}$ y Germán Arellano Cruz $^{2}$

\section{Resumen}

Se realizaron comparaciones entre series temporales de diversidad de especies y de diversidad de familias, con el fin de demostrar que las mediciones de diversidad a partir de un nivel taxonómico superior al de especies, pueden brindarnos series temporales con tendencias equivalentes. Los datos empleados fueron las abundancias de coleópteros colectados con trampas pitfall entre Febrero de 1998 y Febrero del 2001 en la Reserva Nacional de Lachay. Las mediciones de diversidad se hicieron empleando el índice de Shannon, considerando dos niveles espaciales distintos (diversidades alfa y gamma). En todos los casos, se hallaron coeficientes de correlación y regresión altamente significativos entre las series temporales de diversidad, asimismo los intervalos de confianza de la diversidad alfa promedio mostraron un alto grado de traslape para la mayoría de fechas de evaluación. Estos resultados respaldan el uso de la diversidad de familias como una alternativa útil, para evitar el esfuerzo ligado a la determinación taxonómica de las especies, en prácticas de manejo ambiental.

Palabras clave: Diversidad, Índice de Shannon, metodología para manejo ambiental, Coleoptera, Reserva Nacional de Lachay.

\begin{abstract}
Comparisons between temporal series of specific and family diverstity were made to demonstrate that diversity measurements at a higher taxonomic level that species, can give to us temporal series with equivalent tendencies. The data employed were the abundances of beetles (Coleoptera) collected with pitfall traps from February 1998 to February 2001, at the Lachay National Reserve. The diversity measurements were made with the Shannon's index, considering two spacial levels (alfa and gamma diversities). The correlation and regression coefficients between temporal diversity series had a high level of significance for the all cases, also the confidence intervals for the average alfa diversity show a high degree of intersection for the majority of evaluation dates. These results support the use of the family diversity as a useful choice, to avoid the effort of the taxonomic determination of species, in environmental management practices.
\end{abstract}

Key words: Diversity, Shannon's index, environmental management methodology, Coleoptera, Lachay National Reserve.

\section{Introducción}

La diversidad ha sido descrita como indicadora de un conjunto de aspectos del funcionamiento de los ecosistemas, tales como la cantidad e intensidad de las interacciones poblacionales que tienen lugar al interior del sistema (Margalef, 1978 y 1980) o; la calidad y cantidad del flujo energético disponible (Odum, 1980). Desde un punto de vista aplicativo, ha sido y es utilizada tanto en el campo de la biología de la conservación como en el de la supervisión ambiental, como un índice de salud del ecosistema. En el campo de la supervisión ambiental se supone que los efectos adversos de la polución se reflejan en una reducción de la diversidad y asimismo, sirve para añadir rigor científico a las decisiones que de otro modo, podrían realizarse exclusivamente bajo puntos de vista subjetivos (Magurran, 1988).

La diversidad basada en el número de especies es la expresión más generalizada de este parámetro, pero tal como lo señala Margalef (1991), ésta plantea por lo menos dos dificultades: Primero, es impensable dar un censo completo, porque continuamente se descubren especies nuevas y segundo, no es posible convocar a los expertos necesarios para censar especies de todos los grupos de organismos. Por lo tanto, ambas dificultades conducen generalmente a restringir las evaluaciones de diversidad a colectivos limitados.

Aún cuando las evaluaciones se restrinjan a una sola comunidad de todas las que integran el ecosistema, el problema de hallar especies nuevas

\footnotetext{
${ }^{1}$ Laboratorio de Control Biológico y Ecología de Artrópodos del Departamento Académico de Biología. Universidad Nacional Agraria La Molina. Av. La Universidad S/N. Apartado 456. Lima 100. Dirección electrónica: ${ }^{1}$ troodon76@hotmail.com/²acg@lamolina.edu.pe
} 
subsiste y esto ha llevado a los investigadores a crear la categoría de "morfoespecies", para designar a especies carentes aún de una descripción formal (Lawton et al., 1998; Werner \& Raffa, 2000) o incluso a individuos inmaduros difíciles de identificar plenamente (Dennis et al., 1998). A pesar de los beneficios prácticos obtenidos al trabajar con morfoespecies, a medida que se reduce el tamaño corporal de los taxa examinados, se registra un aumento en la proporción de las mismas que incrementa significativamente el número de horas de trabajo requeridas para procesar las muestras (Lawton et al., 1998).

En el campo de la biología de la conservación se ha planteado la posibilidad de reemplazar las mediciones tradicionales de biodiversidad a través de la riqueza de especies, por otras basadas en la riqueza de taxones superiores, con el fin de acelerar las investigaciones y reducir sus costos. Williams \& Gaston (1994), demostraron que la riqueza de familias es un buen predictor de la riqueza de especies, para una amplia variedad de grupos taxonómicos y regiones del planeta. La riqueza de familias ha sido empleada satisfactoriamente para la ubicación de los centros de biodiversidad mundial de las plantas angiospermas (Williams et al., 1994) y ha mostrado patrones coincidentes para la distribución mundial de la biodiversidad entre angiospermas, anfibios, reptiles y mamíferos (Williams et al., 1997).

Como parte de las bases metodológicas del Proyecto Iberoamericano de Biogeografía y Entomología Sistemática (PRIBES) se exploró la relación entre el número de especies y el número de taxones de alto rango (géneros y familias) en casos concernientes a varios grupos de artrópodos terrestres (Martín Piera, 2000), comprobando que este tipo de funciones proporciona predicciones congruentes con otras estimaciones de riqueza máxima dependiendo de la intensidad del muestreo, de la escala espacial a la que se realiza el inventario y de la "distancia" taxonómica entre variables. Martín Piera (2000) resalta el valor y la atención que deberían merecer estas aproximaciones metodológicas, frente a la ausencia de inventarios exhaustivos para casi la totalidad de grupos de insectos en las diferentes regiones del Planeta.

El propósito del presente trabajo es demostrar que las mediciones de diversidad empleando índices no paramétricos (el índice de Shannon en este caso) pueden brindarnos series temporales equivalentes, no obstante se sustituya en su cálculo a la especie por el nivel taxonómico de familia. Por tanto, se trata de fundamentar una propuesta metodológica de sustitución para la diversidad específica, análoga a la que viene siendo desarrollada en la biología de la conservación (Williams \& Gaston, 1994 ; Williams et al., 1994 ; Williams et al., 1997 ; Martín Piera, 2000), para aplicarse en el campo del manejo ambiental.

\section{Materiales y Métodos}

Se emplearon los datos de abundancia de los coleópteros colectados con trampas pitfall, en 13 muestreos efectuados entre Febrero de 1998 y Febrero del 2001 en la Reserva Nacional de Lachay $\left(11^{\circ} 21^{\prime} 00^{\prime \prime}-11^{\circ} 21^{\prime} 58^{\prime \prime}\right.$ L.S. y $77^{\circ} 22^{\prime} 25^{\prime \prime}$ $77^{\circ} 22^{\prime} 28^{\prime \prime}$ L.O.). Los detalles del área de estudio, la metodología de muestreo, el trabajo de laboratorio y la determinación taxonómica, pueden ser consultados en Giraldo (en preparación).

\section{Cálculo de la diversidad}

Para calcular la diversidad de especies y familias, se empleó el índice de diversidad de Shannon (Magurran, 1988). Este método basado en la teoría de la información es uno de los más usados para el cálculo de este parámetro (Magurran, 1988; Krebs, 1989):

$$
H=-\underset{i=1}{S}\left(p_{i}\right)\left(\log _{2} p_{i}\right)
$$

Donde:

$\mathrm{H}=$ Información contenida en la muestra (bits / individuo).

$=$ Índice de diversidad de especies.

$\mathrm{S}=$ Número de especies.

$\mathrm{p}_{\mathrm{i}}=$ Proporción total de la muestra perteneciente a la iésima especie.

La diversidad fue calculada para dos niveles espaciales, para cada unidad muestral (diversidad alfa) y para el área de estudio completa (diversidad gamma). Asimismo, se calculó el promedio de la diversidad alfa para cada muestreo. Los intervalos de confianza para este último, se obtuvieron a partir de la distribución $\mathrm{t}$ de Student $(\mathrm{a}=0.05$, g.1.= n-1), según la siguiente expresión (Duncan et al., 1978):

$$
\text { I.C. }=\mathrm{H}_{\text {prom }}^{\prime} \pm \mathrm{t}_{\mathrm{a} / 2}\left(\mathrm{~S} / \mathrm{n}^{1 / 2}\right)
$$

Donde: I.C. $=$ Intervalo de confianza

$\mathrm{H}^{\prime}{ }_{\text {prom }}=$ Promedio de la diversidad alfa

$\mathrm{t}_{\mathrm{a} / 2}=$ Valor tabular de la distribución $\mathrm{t}$

$\mathrm{S}=$ Desviación estándar de la diversidad alfa

$\mathrm{n}=$ número de parcelas

\section{$\underline{\text { Regresiones y correlaciones }}$}

Los parámetros de regresión ( $\mathrm{a}, \mathrm{b})$ y sus respectivos errores estándar, el coeficiente de Pearson $\left(\mathrm{r}^{2}\right)$ y el coeficiente de Spearman (r) se obtuvieron utilizando el programa Statistica versión 6.0.

Las regresiones y correlaciones fueron efectuadas entre las siguientes series de valores de diversidad de especies y diversidad de familias: 
1. Los valores de diversidad alfa obtenidos durante cada muestreo. Un total de 13 series con 31 valores como máximo para cada una.

2. Los valores de diversidad alfa promedio correspondientes a los 13 muestreos.

3. Los valores de diversidad gamma correspondientes a los 13 muestreos.

Los intervalos de confianza para los parámetros de regresión (a y b) se calcularon usando la distribución t, con un $\mathrm{a}=0.05$ y g.l. $=\mathrm{n}-2$, según la siguiente fórmula (Steel y Torrie, 1988):

$$
\text { I.C. }=\text { p.r. } \pm \mathrm{t}_{\mathrm{a} / 2} \cdot \mathrm{S}_{(\mathrm{p} . \mathrm{r})}{ }^{2}
$$

Donde:

p.r.= parámetro de regresión

$\mathrm{t}_{\mathrm{a} / 2}=$ valor tabular de la distribución $\mathrm{t}$

$\mathrm{S}_{(\mathrm{p} . \mathrm{r})}{ }^{2}=$ error estándar del parámetro de regresión

Se realizó una prueba de comparación entre pendientes basada en la distribución $t$, de acuerdo a la siguiente fórmula (Levin y Rubin, 1996):

$$
\mathrm{t}=(\mathrm{b}-\mathrm{Bho}) / \mathrm{S}_{\mathrm{b}}
$$

Donde: $\quad \mathrm{b}=$ pendiente de la regresión ajustada $\mathrm{BHo}=$ pendiente hipotetizada $\mathrm{S}_{\mathrm{b}}=$ error estándar de la pendiente "b"

Los valores calculados se compararon con un $t$ crítico para $\mathrm{n}-2$ y a un nivel de significación de $\mathrm{a}=$ 0.05 .

\section{Resultados y Discusión}

Al examinar la relación entre las diversidades alfa de especies y familias en cada muestreo, se observa que en términos generales ésta es bastante estrecha (Tabla 1). Los coeficientes de correlación y regresión de mayor significación corresponden a los muestreos de Mayo-98 y Diciembre-98 (Figuras 1 y 2), en tanto los de menor significación fueron los obtenidos para los muestreos de Agosto-99 y Febrero-2001 (Figuras 3 y 4). Los intervalos de confianza de los parámetros a y b son traslapantes entre todos los muestreos tal como puede verse en la tabla 2. El grado de traslape y el rango de valores incluido dentro de los intervalos es mucho más constante para el parámetro b que para el parámetro a (Figuras 5 y 6), lo cual nos indica que en la mayoría de los casos, existe una proporción establecida entre los valores de ambas diversidades, pero a la vez una amplia gama de posibles valores de diversidad de especies para el caso en el cual la diversidad de familias sea igual a cero. Es debido a esta fluctuación en el valor del parámetro a, así como al hallazgo de diferencias significativas entre algunas de las pendientes halladas (Tabla 3), que no resulta pertinente formular una ecuación predictiva.
Las series temporales de diversidad alfa promedio y gamma de familias siguen tendencias que corresponden de modo muy aproximado a las tendencias descritas por las series análogas de diversidad de especies (Figuras 7 y 8 ). Dichos comportamientos equivalentes se ven reflejados en los altos coeficientes de correlación y regresión obtenidos, los cuales son más fuertes entre las series temporales de diversidad gamma $\left(r=0.952, r^{2}=0.906\right)$, que entre las de diversidad alfa promedio $\left(\mathrm{r}=0.917, \mathrm{r}^{2}=0.841\right)$. En ambos casos, los coeficientes de regresión obtenidos fueron de una muy alta significación estadística, $\mathrm{F}_{\mathrm{ANVA}}=105.738 \quad(\mathrm{a}=0.05, \mathrm{p}<0.001)$ entre diversidades gamma y $\quad \mathrm{F}_{\mathrm{ANVA}}=58.154 \quad(\mathrm{a}=0.05$, $\mathrm{p}<0.001)$ entre diversidades alfa promedio. Tal grado de significación, también puede apreciarse visualmente por la escasa dispersión que presentan sus puntos (Figuras 9 y 10). No sólo las tendencias tuvieron una alta correspondencia, sino que además, los valores de diversidad obtenidos fueron muy cercanos, a tal punto que como se observa en la Figura 11, los intervalos de confianza para la diversidad alfa promedio se traslapan en la mayoría de ocasiones.

De acuerdo a los resultados obtenidos, prácticas de manejo ambiental que involucren mediciones de diversidad, tales como evaluaciones de impacto ambiental y monitoreos, podrían realizarse sin la necesidad de un trabajo tan exhaustivo, como el que se requiere para lograr la determinación de especies. Sin embargo, no sabemos hasta qué punto nuestros resultados pueden ser extrapolados a comunidades correspondientes a otros taxas y conformantes de ecosistemas distintos al de las lomas. Al respecto, cabe señalar tres atributos de la comunidad examinada: Primero, pertenece al orden Coleoptera, el más amplio del Reino Animal (Britton, 1973) y por tanto, la probabilidad de hallar un gran número de familias es elevada (21 en el presente caso); segundo, la proporción de familias representadas por una sola especie en este caso es bastante alta (48 \%) y tercero; la diversidad de esta comunidad es fuertemente dependiente de la equidad, debido al predominio de distribuciones de abundancia con dominancias marcadas (Giraldo, en prep.).

Mientras que los dos primeros atributos señalados resultan benéficos para la aplicación de nuestra propuesta metodológica, por su parte la influencia de la equidad en el comportamiento de la diversidad conduce a toda una gama de situaciones. Una disminución de la equidad será benéfica para la relación entre diversidades cuando la especie dominante es el único representante de su familia, como ocurrió durante Mayo-98 (Figura 1) con Pterostichus sp. de la familia Carabidae. En cambio, una disminución de equidad debida a la dominancia conjunta de varias especies de la misma familia resulta perjudicial para la correspondencia entre las diversidades, tal como sucedió con la dominancia de 
la familia Tenebrionidae durante Febrero-2001 (Figura 4). Un aumento de equidad como el registrado en Agosto-99 (Figura 3) debilita la relación entre diversidades, debido al aumento en el valor de la diversidad específica. Si consideramos un valor de equidad intermedio entre los observados en la presente investigación, notamos una alta significación en la relación obtenida para Diciembre-98 (Figura 2). Es conveniente anotar que el efecto de la equidad sobre la relación entre diversidades es claro sólo al tratarse los casos extremos, mientras que los casos intermedios, responden mas bien a sus combinaciones particulares de equidad y riqueza.

En virtud de los atributos señalados y en ausencia de un análisis comparativo, nuestra propuesta metodológica de sustituir la diversidad de especies por la diversidad de familias se limita por el momento a comunidades pertenecientes a grupos taxonómicos amplios, en las que gran parte de las familias estén representadas por una sola especie y cuya diversidad esté regida por la equidad, siempre guardando la debida precaución frente a la dominancia de una familia, la misma que puede ser típica de alguna temporada del año.

La ausencia de una ecuación predictiva que permita estimar la diversidad específica a partir de la diversidad de familias, parece ser uno de los puntos débiles de nuestra propuesta. No obstante, no la consideramos una grave objeción, por cuanto nuestro propósito se centra en la interpretación que podamos obtener de las series temporales de diversidad y no en los valores obtenidos.

Al comparar nuestra propuesta, con las realizadas en el ámbito de la biología de la conservación (Williams \& Gaston, 1994; Williams et al., 1994 ; Williams et al., 1997; Martín Piera, 2000), destacan dos diferencias notables: primero, las mediciones son de riqueza, por lo tanto no se tiene valores cero y segundo, las unidades espaciales empleadas son del orden de miles de $\mathrm{m}^{2}$, por lo cual resulta poco probable que se produzca la predominancia de una sola familia.

\section{Conclusiones}

-La diversidad de familias presenta series temporales con tendencias equivalentes a las de la diversidad de especies.

-La diversidad de familias, tomando en cuenta las restricciones señaladas, podría sustituir a la diversidad de especies en las prácticas de manejo ambiental que requieran el cálculo de dicho parámetro.

\section{Agradecimientos}

Los autores agradecen al Consejo Nacional de Ciencia y Tecnología (CONCYTEC), por el apoyo financiero dado para la realización de las salidas de campo. Al personal de la Reserva Nacional de Lachay por las facilidades brindadas durante cada uno de los muestreos. A la Dra. Consuelo Arellano por las correcciones y valiosas sugerencias hechas al manuscrito original. De manera especial, al Dr. Edgar Sánchez Infantas por sugerir el tema y motivar el desarrollo del presente artículo.

\section{Referencias bibliográficas}

Britton E.B. 1973. Coleoptera (Beetles). En: The Insects of Australia. Melbourne University Press.

Dennis P., Young M.R. \& Gordon I.J. 1998. Distribution and abundance of small insects and arachnids in relation to structural heterogeneity of grazed, indigenous grasslands. Ecological Entomology. 23 (3): 253-264.

Duncan R., Knapp R. y Clinton M. 1978. Bioestadística. Nueva Editorial Interamericana S.A. de C.V. México D.F.

Giraldo A. en prep. Análisis de los patrones de variación espacio-temporal de las poblaciones de coleópteros en la Reserva Nacional de Lachay durante el periodo 1998 - 2001. Tesis para optar el Título de Biólogo - Universidad Nacional Agraria La Molina.

Krebs J. 1989. Ecological Methodology. University of British Columbia. Harper Collins Publishers.

Lawton J.H., Bignell D.E., Bolton B., Bloemers G.F., Eggleton P., Hammond P.M., Hodda M., Holt R. D., Larsen T.B., Mawdsley N.A., Stork N.E., Srivastava D.S. \& Watt A.D. 1998. Biodiversity inventories, indicator taxa and effects of habitat modifications in tropical forest. Nature 391: 72-76.

Levin R. y Rubin D. 1996. Estadística para administradores. Prentice-Hall Hispanoamericna S.A. Naucalpan de Juárez México.

Magurran A. 1988. Diversidad Ecológica y su Medición. Ediciones Vedra S.A.

Margalef R. 1978. Perspectivas de la teoría ecológica. Editorial Blume. Barcelona-España.

1980. Diversidad, estabilidad y madurez en los ecosistemas naturales. En: Conceptos unificadores en ecología. Edit. Blume BarcelonaEspaña.

1991. Reflexiones sobre la Diversidad y significado de su expresión cuantitativa. Diversidad Biológica: 105-114. Fundación Araces, Madrid.

Martín Piera F. 2000. Estimaciones prácticas de biodiversidad utilizando taxones de alto rango en insectos. Proyecto Iberoamericano de Biogeografía y Entomologia Sistematica. Resumen disponible en http://entomologia.rediris.es/pribes

Odum E. 1980. La Diversidad como función del flujo de energía. En: Conceptos unificadores en ecología. Edit. Blume Barcelona-España.

Steel R. y Torrie J. 1988. Bioestadística: Principios y procedimientos. Mc Graw-Hill / Interamericana de México S.A. de C.V. 
Werner S.M. \& Raffa K.F. 2000. Effects of forest management practices on the diversity of groundocurring beetles in mixed northern hardwood forest of the Great Lakes Region. Forest Ecology and Management. 139: 135-155.

Williams P.H., Humphries C.J. \& Gaston K.J. 1994. Centres of seed-plant diversity: the family way. Proceedings of the Royal Society, Biological Sciences. 256: 67-70. Abstract disponible en http://www.nhm.ac.uk/science/projects/worldmap/ diversity/ htaxa.htm

Williams P.H. \& Gaston K.J. 1994. Measuring more of biodiversity: can higher-taxon richness predict wholesale species richness? Biological Conservation. 67: 211-217. Abstract disponible en http://www.nhm.ac.uk/science/projects/worldmap/ diversity/htaxa.htm

Williams P.H., Gaston K.J. \& Humphries C.J. 1997. Mapping biodiversity value worldwide: combining higher-taxon richness from different groups. Proceedings of the Royal Society, Biological Sciences. 264: 141-148. Abstract disponible en http://www.nhm.ac.uk/science/projects/worldmap/ diversity/ htaxa.htm

Anexos: Tablas y figuras citadas en el texto en las siguientes páginas.
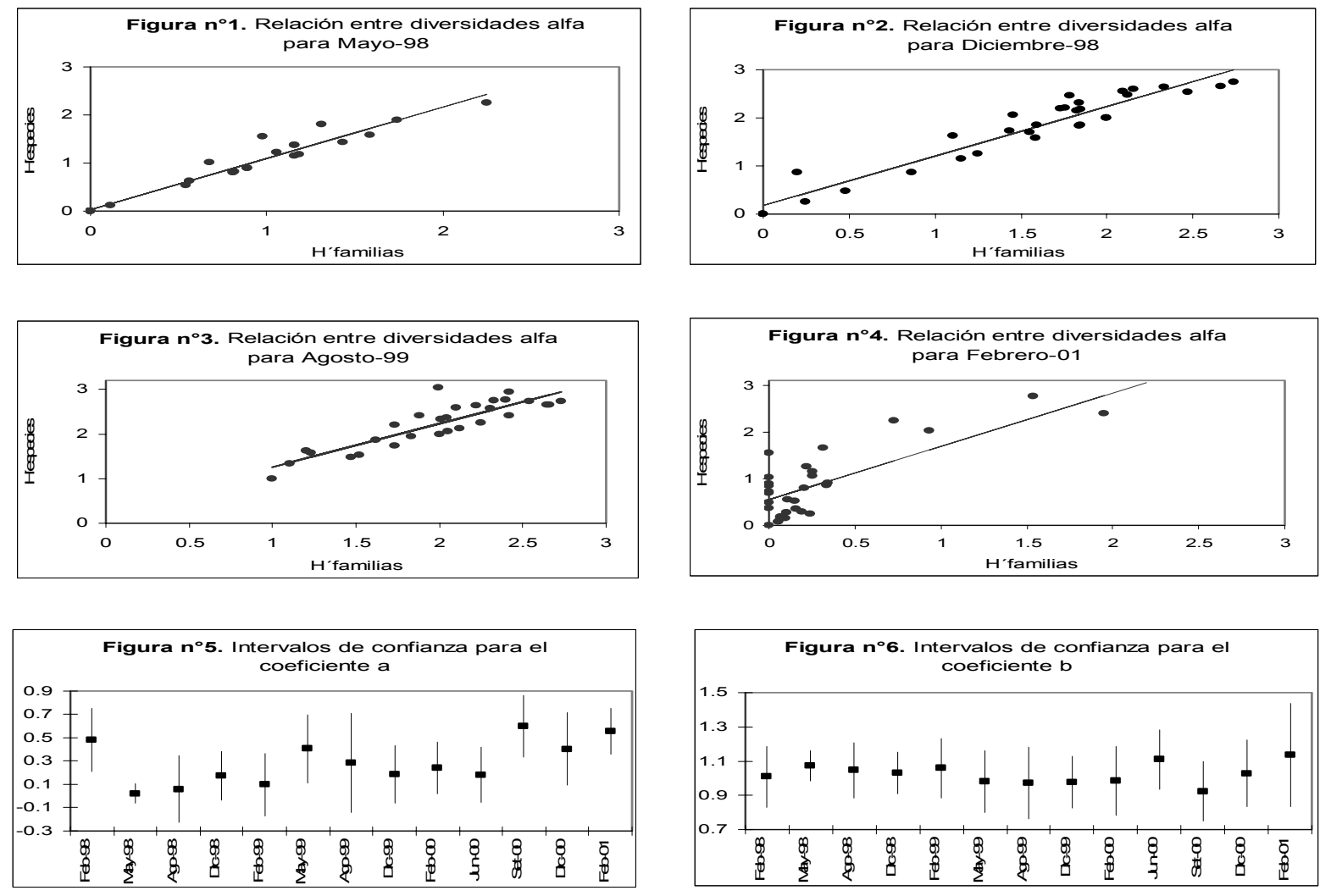
Diciembre 2002
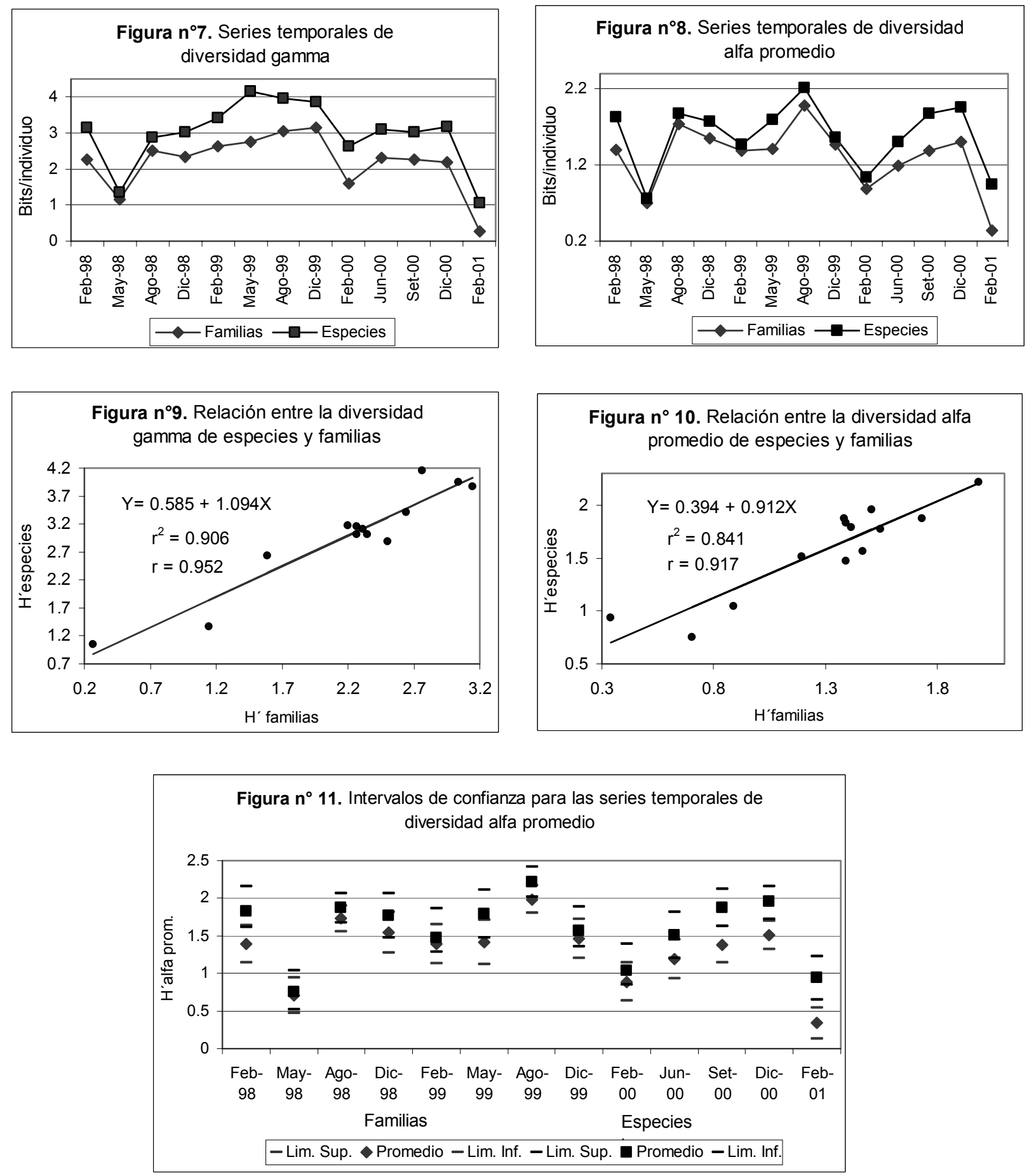


\section{ALFREDO GIRALDO Y GERMÁN ARELLANO}

Ecología Aplicada, Vol. 1, No 1, pp. $43-49$

Tabla $\mathbf{n}^{\circledR}$ 1. Coeficientes de correlación y regresión, parámetros de regresión y valores de $\mathrm{F}_{\text {ANVA }}$ para la relación entre la diversidad alfa de especies y familias en cada uno de los muestreos

\begin{tabular}{|c|c|c|c|c|c|}
\cline { 2 - 6 } \multicolumn{1}{c|}{} & $\mathbf{r}$ & $\mathbf{r}^{2}$ & $\mathbf{a}$ & $\mathbf{b}$ & ${ }^{*} \mathbf{F}$ \\
\hline Feb-98 & 0.912 & 0.832 & 0.484 & 1.008 & 138.237 \\
\hline May-98 & 0.978 & 0.957 & 0.023 & 1.073 & 621.047 \\
\hline Ago-98 & 0.929 & 0.864 & 0.06 & 1.046 & 177.516 \\
\hline Dic-98 & 0.955 & 0.912 & 0.175 & 1.03 & 301.185 \\
\hline Feb-99 & 0.926 & 0.857 & 0.098 & 1.059 & 155.475 \\
\hline May-99 & 0.9 & 0.811 & 0.405 & 0.979 & 124.124 \\
\hline Ago-99 & 0.879 & 0.773 & 0.285 & 0.973 & 91.806 \\
\hline Dic-99 & 0.928 & 0.861 & 0.186 & 0.977 & 173.723 \\
\hline Feb-00 & 0.892 & 0.796 & 0.242 & 0.986 & 101.158 \\
\hline Jun-00 & 0.926 & 0.858 & 0.183 & 1.11 & 169.722 \\
\hline Set-00 & 0.902 & 0.813 & 0.597 & 0.923 & 117.704 \\
\hline Dic-00 & 0.895 & 0.801 & 0.404 & 1.028 & 116.709 \\
\hline Feb-01 & 0.819 & 0.67 & 0.554 & 1.135 & 58.976 \\
\hline
\end{tabular}

${ }^{*} F_{\text {ANVA }}: a=0.05, p<0.001$

Tabla $\mathbf{n}^{\circ}$ 2. Intervalos de confianza para los parámetros de regresión a y b

\begin{tabular}{|c|c|c|c|c|}
\cline { 2 - 5 } \multicolumn{1}{c|}{} & \multicolumn{2}{c|}{ a } & \multicolumn{2}{c|}{ b } \\
\cline { 2 - 5 } \multicolumn{1}{c|}{} & Lim.Inf. & Lim.Sup. & Lim.Inf. & Lim.Sup. \\
\hline Feb-98 & 0.21366 & 0.75434 & 0.83187 & 1.18413 \\
\hline May-98 & -0.061 & 0.10697 & 0.98494 & 1.16106 \\
\hline Ago-98 & -0.2288 & 0.34877 & 0.88421 & 1.20779 \\
\hline Dic-98 & -0.0336 & 0.38359 & 0.90935 & 1.15066 \\
\hline Feb-99 & -0.1713 & 0.36734 & 0.88424 & 1.23376 \\
\hline May-99 & 0.11461 & 0.69539 & 0.79904 & 1.15896 \\
\hline Ago-99 & -0.1398 & 0.70976 & 0.7637 & 1.1823 \\
\hline Dic-99 & -0.0598 & 0.43176 & 0.82545 & 1.12855 \\
\hline Feb-00 & 0.01995 & 0.46405 & 0.78451 & 1.18749 \\
\hline Jun-00 & -0.0566 & 0.42262 & 0.93592 & 1.28408 \\
\hline Set-00 & 0.33229 & 0.86171 & 0.74858 & 1.09742 \\
\hline Dic-00 & 0.09521 & 0.7128 & 0.83373 & 1.22228 \\
\hline Feb-01 & 0.35768 & 0.75032 & 0.83234 & 1.43766 \\
\hline
\end{tabular}

Tabla $\mathbf{n}^{\circ}$ 3. Matriz de valores obtenidos en las pruebas de comparación entre pendientes

\begin{tabular}{|c|c|c|c|c|c|c|c|c|c|c|c|c|c|}
\cline { 2 - 14 } \multicolumn{1}{c|}{} & Feb-98 & May-98 & Ago-98 & Dic-98 & Feb-99 & May-99 & Ago-99 & Dic-99 & Feb-00 & Jun-00 & Set-00 & Dic-00 & Feb-01 \\
\hline Feb-98 & 0 & -0.7558 & -0.4419 & -0.2558 & -0.593 & 0.33721 & 0.40698 & 0.36047 & 0.25581 & -1.186 & 0.98837 & -0.2326 & -1.4767 \\
\hline May-98 & 1.51163 & 0 & 0.62791 & 1 & 0.32558 & $\mathbf{2 . 1 8 6 0 5}$ & $\mathbf{2 . 3 2 5 5 8}$ & $\mathbf{2 . 2 3 2 5 6}$ & 2.02326 & -0.8605 & $\mathbf{3 . 4 8 8 3 7}$ & 1.04651 & -1.4419 \\
\hline Ago-98 & 0.48101 & -0.3418 & 0 & 0.20253 & -0.1646 & 0.8481 & 0.92405 & 0.87342 & 0.75949 & -0.8101 & 1.55696 & 0.22785 & -1.1266 \\
\hline Dic-98 & 0.37288 & -0.7288 & -0.2712 & 0 & -0.4915 & 0.86441 & 0.9661 & 0.89831 & 0.74576 & -1.3559 & 1.81356 & 0.0339 & -1.7797 \\
\hline Feb-99 & 0.6 & -0.1647 & 0.15294 & 0.34118 & 0 & 0.94118 & 1.01176 & 0.96471 & 0.85882 & -0.6 & 1.6 & 0.36471 & -0.8941 \\
\hline May-99 & -0.3295 & -1.0682 & -0.7614 & -0.5795 & -0.9091 & 0 & 0.06818 & 0.02273 & -0.0795 & -1.4886 & 0.63636 & -0.5568 & -1.7727 \\
\hline Ago-99 & -0.3431 & -0.9804 & -0.7157 & -0.5588 & -0.8431 & -0.0588 & 0 & -0.03922 & -0.1275 & -1.3431 & 0.4902 & -0.5392 & -1.5882 \\
\hline Dic-99 & -0.4189 & -1.2973 & -0.9324 & -0.7162 & -1.1081 & -0.027 & 0.05405 & 0 & -0.1216 & -1.7973 & 0.72973 & -0.6892 & $\mathbf{- 2 . 1 3 5 1}$ \\
\hline Feb-00 & -0.2245 & -0.8878 & -0.6122 & -0.449 & -0.7449 & 0.07143 & 0.13265 & 0.09184 & 0 & -1.2653 & 0.64286 & -0.4286 & -1.5204 \\
\hline Jun-00 & 1.2 & 0.43529 & 0.75294 & 0.94118 & 0.6 & 1.54118 & 1.61176 & 1.56471 & 1.45882 & 0 & $\mathbf{2 . 2}$ & 0.96471 & -0.2941 \\
\hline Set-00 & -1 & -1.7647 & -1.4471 & -1.2588 & -1.6 & -0.6588 & -0.5882 & -0.63529 & -0.7412 & $\mathbf{- 2 . 2}$ & 0 & -1.2353 & $\mathbf{- 2 . 4 9 4 1}$ \\
\hline Dic-00 & 0.21053 & -0.4737 & -0.1895 & -0.0211 & -0.3263 & 0.51579 & 0.57895 & 0.53684 & 0.44211 & -0.8632 & 1.10526 & 0 & -1.1263 \\
\hline Feb-01 & 0.85811 & 0.41892 & 0.60135 & 0.70946 & 0.51351 & 1.05405 & 1.09459 & 1.06757 & 1.00676 & 0.16892 & 1.43243 & 0.72297 & 0 \\
\hline
\end{tabular}

